\title{
Influência dos fotopolimerizadores (luz halógena X led) na resistência à compressão de resinas compostas
}

\author{
Halogen curing light $X$ led influence on compression resistence of composite resin
}

\section{Danielle Figueiredo Accetta'; Thalles Ribeiro Magalhães Filho²; Karin de Mello Weig ${ }^{3}$; Ricardo Carvalhaes Fraga ${ }^{4}$,}

${ }^{1}$ Especialista em Prótese dentária, Mestranda em Clínica Odontológica.

\section{Resumo}

O objetivo deste estudo foi verificar a resistência à compressão de três compósitos usados em restaurações diretas (Surefil - Dentsply; Concept - Vigodent; Esthetx - Dentsply) polimerizados através de dois tipos de aparelhos fotopolimerizadores: um de luz halógena (Gnatus) e outro de diodo emissor de luz (LED) (Gnatus). Foram confeccionados 30 corpos de prova, sendo 10 para cada material, cada um com $4 \mathrm{~mm}$ de diâmetro por $6 \mathrm{~mm}$ de comprimento. Cinco corpos de prova de cada material foram polimerizados com luz halógena e cinco com LED. A manipulação e a polimerização dos materiais foram feitas seguindo as instruções dos fabricantes. Após a confecção, os corpos de prova foram levados à máquina de ensaio (Instron 4204) com carga de $5 \mathrm{KN}$ e velocidade de $1,0 \mathrm{~mm} / \mathrm{min}$ para o ensaio da resistência à compressão. Os resultados foram submetidos à análise estatística (ANOVA) e indicaram não haver diferença estatisticamente significante entre as médias.

Palavras-chave: Resinas compostas; Materiais dentários.

\section{Abstract}

The purpose of this study was to verify the compression resistance of three composites used in direct restorations (Surefil - Dentsply; Concept - Vigodent; Esthetx - Dentsply) polymerized through two types of curing lights: halogen (Gnatus) and light emitted diode (LED) (Gnatus). We made 30 bodies of evidence, 10 for each material, with $4 \mathrm{~mm}$ in diameter by $6 \mathrm{~mm}$ in length. Five bodies of evidence for each material were polymerized with halogen light and five with LED. The polymerization and manipulation of materials were made following the instructions of manufacturers. After confection, the bodies of evidence were taken from machine test (Instron 4204) with load of $5 \mathrm{KN}$ and speed of $1.0 \mathrm{~mm} / \mathrm{min}$ for the test of compression resistance. The results were subjected to statistical analysis (ANOVA) and showed no statistically significant difference between mean.

Keywords: Resins composites, Dental materials.

\section{Introdução}

Um dos aspectos que contribuem para o sucesso de restaurações de resinas compostas é a polimerização adequada das mesmas, o que depende diretamente da intensidade de luz dos aparelhos fotopolimerizadores. Existem vários tipos de aparelhos para realizar essa fotopolimerização, entre eles estão os aparelhos à base de luz halógena e os diodos emissores de luz (LEDs).

Unidades de luz halógena têm efetividade comprovada e são de custo relativamente baixo. No entanto, esses aparelhos podem apresentar problemas com sua durabilidade e com a emissão de calor. Seu tempo de vida útil é de 40 horas, ou seja, de 6 a 12 meses, dependendo da frequência e dos ciclos de "acende-apaga" aos quais é submetida (VIEIRA et al., 2000; KREIDLER et al., 2004).

Aparelhos de luz halógena apresentam algumas vantagens como tecnologia de baixo custo, boa intensidade de potência e emitem luz num espectro mais amplo. Porém algumas desvantagens também estão presentes como requererem manutenção, gerarem altas temperaturas e possuírem filtros redutores (MACHADO et al., 2007).
${ }^{2}$ Mestre em Engenharia dos Materiais, Doutorando em Engenharia dos Materiais; Professor da Disciplina de Materiais Dentários da UFF.

${ }^{3}$ Mestre em Engenharia dos Materiais, Doutorando em Engenharia dos Materiais; Professor da Disciplina de Materiais Dentários da UFF.

${ }^{4}$ Doutor em Odontologia, Coordenador do Curso de Mestrado em Clínica Odontológica da UFF.

Instituição: Universidade Federal Fluminense (UFF)

Ao contrário das lâmpadas halógenas que geram luz por filamentos incandescentes, os LEDs convertem energia elétrica diretamente em luz por eletroluminescência, o que proporciona um mínimo aquecimento. Sua vida útil é relativamente longa (mais de 10000 horas) e passa por pequena degradação com o seu uso através do tempo (WILLIAN, 2002). Por trabalharem com baixa voltagem, os LEDs podem ser usados com baterias recarregáveis, o que os torna bastante práticos. Eles também são resistentes a choques e vibrações.

Aparelhos a base de LEDs apresentam vantagens como não emitirem radiação infravermelha para a resina composta e para o dente, produzirem pouco calor, reduzindo assim a possibilidade de degradação dos componentes internos ao longo do tempo e não necessitarem de filtros devido ao estreito espectro de comprimento de onda emitido, que coincide com a região de absorção da canforoquinona (fotoiniciador presente nas resinas compostas) (GODOY et al., 2007).

O estudo teve como objetivo avaliar qual desses aparelhos (luz halógena e LED) apresenta melhores resultados quanto à resistência à compressão de três resinas compostas (Concept, Esthetx e Surefil). Para isso, foi utilizada a análise de variância (ANOVA).

\section{Materiais e Métodos}

Foram confeccionados 30 corpos de prova (CP), sendo 10 da resina Surefil, 10 da Concept e 10 da Esthetx, sendo 5 corpos de prova de cada resina polimerizados com LED de sete diodos (Gnatus) e 5 com a luz halógena (Gnatus).

Os corpos de prova para avaliação da resistência à compressão foram confeccionados de acordo com a especificação $n^{\circ} 27$ da ADA. Inicialmente, confeccionou-se cinco matrizes metálicas cilíndricas de $4 \mathrm{~mm}$ de diâmetro e $6 \mathrm{~mm}$ de comprimento, como mostra a Figura 1. As superfícies internas das matrizes foram polidas com lixa de granulação 600 e 1200 para permitir a remoção dos corpos de prova com maior facilidade e dar às superfícies laterais dos mesmos um melhor acabamento.

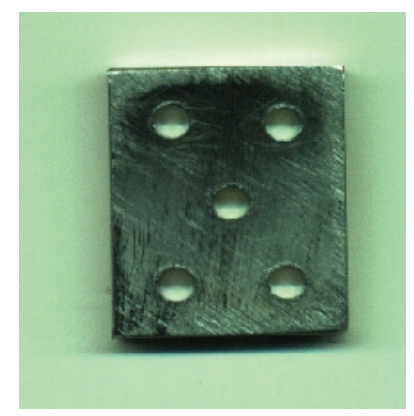

Figura 1. Matriz metálica utilizada para confecção dos corpos de prova. 
O material foi então vazado até o completo preenchimento das matrizes. Em cada uma das superfícies das matrizes foi colocada uma tira de poliéster e uma placa de vidro. Antes da emissão de luz (fotopolimerização) os CP sofreram uma pequena pressão, com a placa de vidro, para remoção do excesso e manutenção da superfície plana. A fotopolimerização foi realizada em cada uma das duas pontas por 60 segundos. Após esse tempo, a matriz foi aquecida em estufa a aproximadamente $200^{\circ} \mathrm{C}$ por 30 segundos para provocar uma pequena dilatação e permitir a remoção do CP com facilidade e sem danos. Convém salientar que todos os corpos de prova sofreram o mesmo aquecimento pela mesma temperatura e pelo mesmo tempo.

Antes da realização dos ensaios, o diâmetro e o comprimento de cada amostra foram medidos com um micrômetro PEACKOC de precisão $0,01 \mathrm{~mm}$. As bases metálicas do dispositivo foram forradas com uma película de teflon para diminuir o atrito com a superfície do compósito durante a compressão.

O teste de resistência à compressão foi realizado em uma máquina Instron 4204, mostrada na Figura 2, com célula de carga de $5 \mathrm{KN}$ e velocidade de $1,0 \mathrm{~mm} / \mathrm{min}$.

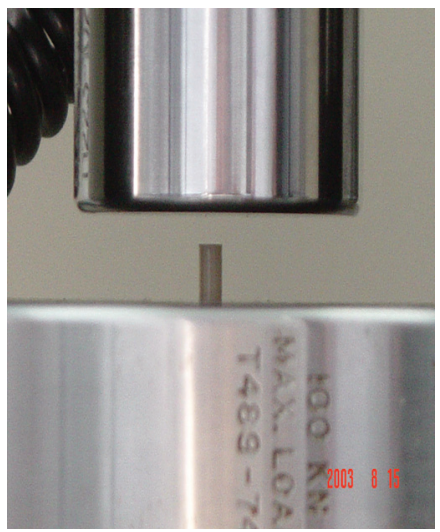

Figura 2. CP posicionado ao centro das bases metálicas do dispositivo, pronto para o prosseguimento do teste de compressão.

Para o cálculo da resistência à compressão, $\sigma_{c}$, foi utilizada a seguinte equação:

$$
\sigma c=\frac{F}{3,14 d^{2} / 4}
$$

Onde $\mathrm{F}=$ Força máxima em Newton exercida $\mathrm{d}=$ diâmetro da amostra em metros

\section{Resultados}

Através da análise estatística de variância (ANOVA), verificou-se que a resistência à compressão dos compósitos polimerizados por luz halógena foram estatisticamente iguais aos seus correspondentes polimerizados por LED.

As resinas compostas Esthetx e Surefil, quando polimerizados por LED, possuíram a resistência à compressão estatisticamente iguais, porém foram mais resistentes que o Concept polimerizado por LED.

Esses resultados podem ser observados através da Tabela 1 e do Gráfico 1.
Tabela 1. Média da resistência à compressão e desvio padrão para as três resinas compostas avaliadas (letras iguais correspondem à igualdade estatística).

\begin{tabular}{lcccc}
\hline \multirow{2}{*}{ Resinas } & \multicolumn{3}{l}{ LUZ HALÓGENA } & \\
\cline { 2 - 5 } & Média (MPa) & Desvio.Padrão & Média (MPa) & Desvio.Padrão \\
\hline Concept & $141,98(\mathrm{a}, \mathrm{d})$ & 46,24 & $123,46(\mathrm{a})$ & 13,73 \\
Esthetx & $197,66(\mathrm{~b}, \mathrm{~d})$ & 69,20 & $238,3(\mathrm{~b})$ & 80,13 \\
& & & & \\
Surefil & $197,68(\mathrm{c}, \mathrm{d})$ & 67,73 & $222,8(\mathrm{c}, \mathrm{b})$ & 44,44 \\
\hline
\end{tabular}

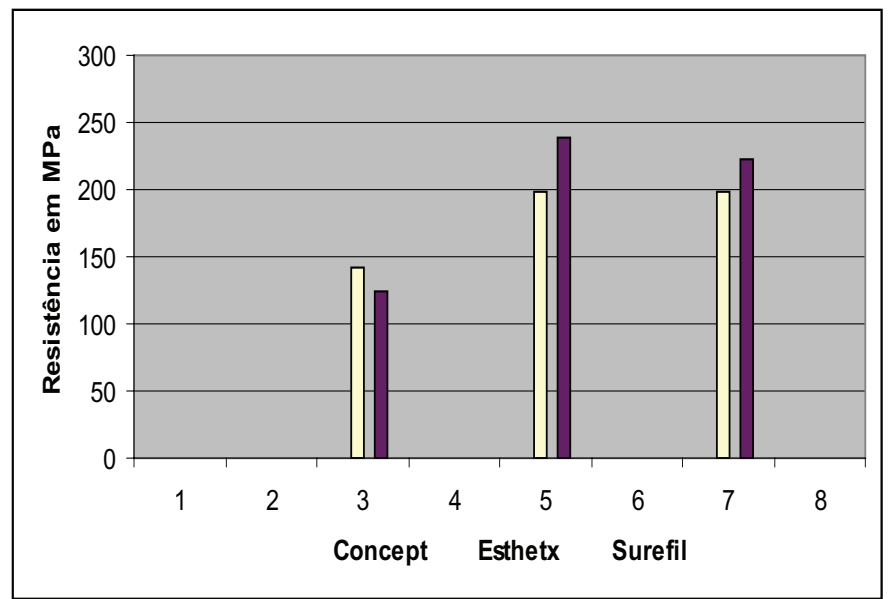

Luz Halógena

LED

Gráfico 1. Média das resistências à compressão para as três resinas compostas avaliadas.

\section{Discussão}

Muitos fatores podem influenciar nas propriedades dos compósitos fotopolimerizados, por exemplo, o comprimento de onda da fonte de luz, o tempo de polimerização e o gradiente de temperatura gerada durante a reação de polimerização. Esses fatores influenciam fortemente o grau de conversão da resina e a obtenção de ótimas propriedades físico-químicas (CONTI et al., 2005).

A fonte de luz mais popular entre os profissionais da área odontológica ainda é a convencional de luz halógena, porém ela apresenta algumas desvantagens que limitam a sua vida útil. Em consequência da alta temperatura gerada durante a emissão da luz, danos no bulbo, refletor e filtro podem afetar o grau de polimerização do material, sem que o profissional possa perceber. Os aparelhos tipo LEDs não possuem filtros, pois comprimentos de onda inespecíficos não são gerados. Há uma mínima geração de calor e não há degradação do fluxo de luz com o passar do tempo (FRANCO; BOSQUIROLI; LOPES et al., 2003).

Unidades de LEDs podem ser comparáveis ou terem melhores efeitos que unidades de luz halógena, dependendo do tipo de resina usada (JONG et al., 2007). O espectro de emissão de luz halógena é grande (entre 380 e $510 \mathrm{~nm}$, com pico perto de $480 \mathrm{~nm}$ ) (FUJIBAYASHI et al.,1998). O LED produz um espectro de onda restrito (450-490nm) (NEMOTO, 1997). Em regiões mais profundas, onde há uma atenuação da luz, a unidade que emite uma faixa de onda mais específica e com alta potência vai proporcionar maior grau de conversão (OBICl et al., 2006). 
Nesse estudo, verificou-se através da análise estatística que não houve diferença estatisticamente significativa entre os compósitos polimerizados por luz halógena e os seus correspondentes polimerizados por LED.

Esses resultados estão de acordo com a observação de Jandt (2000), que demonstrou que os valores de resistência à compressão não foram significativamente diferentes em compósitos polimerizados por LED e por luz halógena. Também estão de acordo com Stahl et al. (2000), que compararam a resistência à compressão de resinas compostas fotoativadas com um aparelho de luz halógena com um aparelho de 27 LEDs e concluíram que não existem diferenças significativas sobre essa propriedade da resina entre as duas fontes de luz testadas. Maníglia et al. (2007) também não encontraram diferença estatisticamente significante na resistência à compressão das resinas testadas quando fotopolimerizadas com luz halógena e com LED. Já Obici et al. (2005) avaliaram a resistência à compressão de uma resina utilizando diferentes métodos de fotoativação, e, dentre os resultados, o LED apresentou menor valor.

É preciso conhecer bem o aparelho fotopolimerizador para obtenção de uma restauração de boa qualidade. Conforme observado nesta pesquisa, tanto unidades de luz halógena quanto de diodo emissor de luz podem gerar uma polimerização satisfatória.

\section{Conclusão}

Diante da metodologia empregada, pode-se concluir que não houve diferença significante entre os dois aparelhos testados.

Tanto os fotopolimerizadores de LEDs quanto os de luz halógena podem ser usados com sucesso na prática clínica. No entanto, os LEDs apresentam algumas vantagens sobre os aparelhos de luz halógena, como o fato de não se degradarem com o tempo, não precisarem de filtro e poderem ser usados com baterias recarregáveis.

\section{Referências}

CONTI, C. et al. Spectroscopic and Mechanical Properties of Dental Resin Composites Cured with Different Light Sources. J. Mol. Struct., Amsterdam, v. 744-747, p. 641-46, June 2005.

FRANCO, E. B.; BOSQUIROLI, V.; LOPES, L. G. LED - uma Nova Tecnologia para Fotopolimerização. Avaliação com Cimento Ionomérico Modificado por Resina. JBC J. Bras. Clin. Odontol. Integr., Curitiba, v. 7, n. 38, p. 116-118, mar./abr. 2003.

FUJIBAYASHI, F. et al. Newly Developed Curing Unit Using Blue Light Emitting Diodes. Dent. Jpn., Tokyo, v. 34, p. 49-53, 1998.

GODOY, E. P. et al. Aparelhos Fotopolimerizadores: Elevação de Temperatura Produzida por Meio da Dentina e Durante a Polimerização da Resina Composta. Rev. Clin. Pesq. Odontol., Curitiba, v. 3, n. 1, p. 11-20, jan./abr. 2007.

JANDT, K. D. Depth of Cure and Compressive Strength of Dental Composites Cured with Blue Light Emiting Diodes (LEDs). Dent. Mat., Copenhagen, v. 16, n. 1, p. 41-47, Jan. 2000.

JONG, C. G. L. et al. The Effectiveness of Different Polymerization Protocols for Class II Composite Resin Restorations. J. Dent., Kidlington, v. 35, n. 6, p. 513-520, June 2007.
KREIDLER, M. A. et al. Avaliação da Intensidade de Luz de Aparelhos Fotopolomerizadores. JBC J. Bras. Clin. Odontol. Integr., Curitiba, v. 8, n. 45, p. 249-253, maio/jun. 2004.

MACHADO, C. T. et al. Novos Tipos de Fontes Fotopolimerizadoras Existentes no Mercado: Conceitos Atuais. Odontol. Clin.-Cient., Recife, v. 6, n. 3, p. 207-211, jul./set. 2007.

MANíGLIA, A. B. et al. Avaliação da Resistência à Compressão de Resinas Compostas Polimerizadas por LED e Luz Halógena. Disponível em http://www.editorasantos.com.br/canalcientifico. Acesso em ago. 2007.

NEMOTO, R. Effect of Wavelength on Polymerization of Light Cured Resins. Dent. Mater. J., Tokyo, v. 16, n. 1, p. 60-73, June 1997.

OBICl, A. C. et al. Avaliação de Propriedades Mecânicas da Resina Composta Z250 Fotoativada com Diferentes Métodos. J. Appl. Oral Sci., Bauru, v. 13, n. 4, p. 393-398, Oct./Dec. 2005.

et al. Monomer Conversion at Different Dental Composite Dephts Using Six Light Curing Methods. Polymer Testing, Essex, England, v. 25, n. 3, p. 282-288, May 2006.

STAHL, F. et al. Light Emiting Diode (LED) Polymerization of Dental Composites: Flexural Properties and Polymerization Potencial. Biomaterials, Guilford, England, v. 21, n. 13, p. 1379-1385, July 2000.

VIEIRA, G. F. et al. Intensidade de Luz de Aparelhos Fotopolimerizadores Utilizados em Consultórios Particulares. J. Bras. Clin. Odontol., Curitiba, v. 4, n. 22, p. 41-44, 2000.

WILLIAN, J. et al. Uma Comparação da Polimerização de Resinas Compostas de Aparelhos com LEDs e Aparelhos com Luz Halógena. Jada Bras. (Ed. Bras.), São Paulo, v. 5, n. 3, p. 142-148, maio/jun. 2002. 